\title{
The Role of Health Economics and Outcomes Research in Health Care Reform in China
}

\author{
Wannian Liang • Jipan Xie • \\ Hongpeng Fu $\cdot$ Eric Q. Wu
}

Published online: 20 February 2014

(c) The Author(s) 2014. This article is published with open access at Springerlink.com

\section{Introduction}

The health care system in China has been undergoing reforms for the past three decades. New policies and regulations are being developed to better meet the health needs of over a billion people. As reform programs expand, the need for scientific evidence increases, to enable accurate evaluation of the impact of new policies. In this context, health economics and outcomes research (HEOR) will be instrumental to evidence-based decision making and is expected to play a major role in China's health care reform.

\section{Reform of China's Health Care System}

The goal of China's health care reform is to establish a system that provides safe, effective, convenient, and affordable services to meet the basic health care needs of the 1.3 billion people living in the country's rural and urban areas [1]. To reach this goal, much effort has been devoted to setting up a health care infrastructure (provider side) and a public health insurance system (payer side).

W. Liang

Department of Healthcare Reform, Office of State Council Health Reform Leading Group, National Health and Family Planning Commission of the People's Republic of China, Beijing, China

J. Xie · E. Q. Wu ( $₫)$

Analysis Group, Inc., 111 Huntington Ave, 10th Floor, Boston,

MA 02199, USA

e-mail: ewu@analysisgroup.com

H. Fu

Department of Medicine Policy, China National Health

Development and Research Center, Beijing, China
As of 2011, there were 954,389 medical institutions and more than 2.5 million licensed physicians in China, substantial increases over 1978 numbers (170,000 medical institutions and 1.0 million licensed physicians) [2]. The current multi-level health insurance system includes Urban Employee Basic Medical Insurance (UEBMI), covering employed urban residents; Urban Resident Basic Medical Insurance (URBMI), for unemployed urban residents; and the New Rural Cooperative Medical System (NRCMS), for people in rural areas. As of 2011, these three national insurance plans were providing coverage for $95 \%$ of the population in China [3]. NRCMS is the largest health plan, covering 832 million people $-97 \%$ of the rural population [4]. In addition, commercial health insurance plans can be purchased to supplement the national health plans.

Improvements in the health care infrastructure and health insurance coverage have in turn led to better health outcomes in the Chinese population. For example, the average life expectancy has increased from 67.9 in 1981 to 73 in 2005, and the infant mortality rate has declined from 50.2 per 1,000 live births in 1991 to 12.1 per 1,000 live births in 2011 [2].

\section{Current Challenges and Future Directions}

Despite such achievements over the past 30 years, China's current health care system still faces a number of challenges. Among these are regional disparities in resource allocation and health insurance coverage, inadequate government funding of public hospitals, and an inefficient and inadequately monitored pharmaceutical market.

Regional disparities, particularly between urban and rural geographies, are evident on both provider and payer sides, reflecting the imbalance in health care system 
developments between the two regions. In terms of health care resource allocation, the majority of medium-to-large hospitals are still located in urban areas. Access to highquality health care services remains a challenge for rural residents, which account for about half of the total population [2]. Insufficient insurance coverage further impacts access in rural areas, as high out-of-pocket costs for serious and chronic diseases often take a considerable financial toll on rural families.

In both rural and urban areas, public hospitals, which provide $91 \%$ of outpatient and inpatient services in China [2], are caught in a financial squeeze. Government spending on health care has been relatively low for many years, with direct government subsidies accounting for only $9 \%$ of public hospital revenues in 2011 [2]. In addition, the government sets prices for many medical services far below actual costs, to improve affordability of these services; as a result, hospitals have to shoulder the financial loss from providing medical services. To make up for lost revenue, most hospitals rely on prescription drug sales and sophisticated diagnostic procedures, which has led to overprescription of expensive and unnecessary drugs and tests [5]. This not only wastes health care resources, it also imposes a heavy financial burden on patients and payers.

Another issue facing China's health care system is the inefficiency of the pharmaceutical supply chain. Manufacturers have little incentive to produce low-cost, effective drugs due to small profit margins. Drug costs are inflated as a result of the multiple layers of distribution between manufacturers and hospitals. In addition, and of significant concern, drug safety and adverse events remain inadequately monitored and managed, presenting potential public health problems.

The Chinese government has proposed and implemented a series of new reform measures to address these challenges. Efforts to increase public health funds and ensure continuous investment in basic health care infrastructure are likely to help further reduce urban-rural disparities in resource allocation. NRCMS coverage is also being expanded and the benefits of national health insurance plans increased, enabling access to a wider range of health care services, particularly among rural residents [6]. In 2012, the Ministry of Health expanded the national essential drug list (EDL), adding 213 more drugs than those on the 2009 formulary [7]. Drugs on the EDL are available through primary care centers with no mark-up in pricing, therefore expanding the list may further enhance the accessibility and affordability of a broader range of treatments and help narrow the gap in medical services provided to urban and rural areas. In addition, the government is taking steps to limit the financial incentives for hospitals to sell expensive and unnecessary drugs and diagnostic tests by, for example, capping the percentage of total hospital revenues derived from drug sales and experimenting various bundled payment schemes such as capitation payment, single-disease payment and per diem payment [8]. These bundled payments would only reimburse hospitals a fixed amount at patient, disease, or per diem level, thus reducing incentives to prescribe costly medicines or procedures.

\section{Role of Health Economics and Outcomes Research to China's Health Care Reforms}

HEOR has long played an essential role in health policy and health care decision making in many countries [9-13]. In China, with reform measures in place, public health officials have begun to recognize the need to generate scientific evidence on the effects of new policies to further inform their decision making. Health economics has thus attracted more attention in recent years; during the past decade, a growing body of health economics and outcome research literature has emerged from China, e.g. studies assessing disease burden, evaluating health care policies, and comparing interventions in China [14-21]. In addition, to improve research quality and standardize methods, multiple academic groups in China are proactively developing guidelines for health economics research. One such guideline, published in 2011, details the methods and best practices in pharmacoeconomic evaluations [22]. Conceivably, the availability of more HEOR would lead to better policymaking and play a critical role in continuing the advancement of reform efforts.

However, the growth and impact of HEOR is limited by lack of high-quality, reliable data sources. Payers and providers both realize the importance of considering the economic values of alternative treatments when making formulary decisions; however, to date the majority of pharmacoeconomic evaluations are cost-minimization studies that include only drug costs. Lack of evidence of comparative effectiveness and medical cost offsets prevent the appropriate implementation of cost-effectiveness analysis. High-quality longitudinal databases integrating inpatient, outpatient, and pharmacy resource utilization, and including clinical outcomes, are in high demand but have yet to be developed.

On a more positive note, substantial efforts have been devoted to developing and collecting data useful in HEOR. With the development of health informatics systems, many hospitals in China have switched from paper medical charts to electronic medical records (EMR). Recently, large, highquality, electronically-linked databases for research use have started to emerge [23, 24]. These databases feature comprehensive clinical and economic information and large sample sizes, even for rare diseases. Also, with the 
expansion of national health insurance coverage, national and regional insurance claims databases are being constructed and upgraded $[25,26]$. In addition, other national and regional data sources (e.g. surveys) have become increasingly available to researchers [27, 28]. Development of a standard, accurate coding system is an ongoing effort and will improve the utility of these data sources, particularly large EMR and claims databases, in future HEOR efforts.

\section{In this Special Issue}

This special issue of PharmacoEconomics brings together studies addressing aspects of China's current health care system from an HEOR perspective. Several of the studies also highlight areas of particular challenge in the state's health care reform initiatives. One study assesses the extent to which the Essential Medicines Program has promoted rational use of medicine and removed the incentives for primary care institutions to prescribe expensive drugs [29]. The study uses China's National Health Services Survey data and shows that removal of economic incentives alone may not be enough to change the health care providers' behaviors and improve rational use of medicines. However, as the authors suggest, the data period may not be long enough to allow the Essential Medicines Program to take effect. More accurate measures defining 'rational use' and future studies are needed to further address this important question. Another study uses a simultaneous equation modeling approach and finds a positive effect of the URBMI on inpatient health service utilization [28]. It would be interesting to further investigate whether the increase in health services utilization enables patients who could not afford health care to receive appropriate care, or reflects overuse of health care services. Additionally, an empirical study describes pharmaceutical pricing under the interplay of market competition and government control in Chinese hospitals [30]. The study provides a helpful introduction to familiarize readers with the systems of pharmaceutical products' pricing and procurement in China; the findings suggest that the local leading manufacturers may be in a disadvantageous position as a result of market competition and government regulations [30]. Lastly, a 20-year study provides an overview of the trends and factors determining pharmaceutical expenditure [31]. It presents empirical evidence on the increasing trend in pharmaceutical expenditure in China. Although economic growth may check the increase in the ratio between total pharmaceutical expenditure and total health care expenditure, the high ratio indicates that measures need to be taken to further reduce the pharmaceutical expenditure in China [31].
The issue also includes several disease-specific topics. An analysis of treatment for venous thromboembolism based on Chinese EMRs data revealed potential undertreatment, suboptimal outcomes, and substantial economic burden among hospitalized patients with the disease [23]. A study among patients with acute myocardial infarction in Shanghai found the inclusion of heart stents in the insurance benefit did not achieve intended improvement of equality on health care utilization without properly addressing health care providers' incentives [32]. A costutility analysis showed that the adjuvant therapy with capecitabine and oxaliplatin recommended by the Chinese National Comprehensive Cancer Network guidelines was cost-effective for the treatment of resectable gastric cancer patients in the long run but not in the short term, suggesting the importance of considering the long-term economic value of new technology in China's health care system [33].

All of these studies provide interesting findings with implications for policy and medical decision making. As China expands its efforts to reform the national health care system, the need for rigorous, relevant HEOR will grow. The editors of this special issue hope that evidence from these studies will be informative to public health policy makers and help to optimize the allocation and utilization of health care resources in China.

Open Access This article is distributed under the terms of the Creative Commons Attribution Noncommercial License which permits any noncommercial use, distribution, and reproduction in any medium, provided the original author(s) and the source are credited.

\section{References}

1. The Office of the Leading Group for Deepening the Reform of the Medical and Health Care System of the State Council. Q\&A on deepening the medical and health care system reform [in Chinese]. Beijing: National Development and Reform Commission; 2009.

2. Ministry of Health. China public health statistical yearbook 2012 [in Chinese]. http://www.nhfpc.gov.cn/htmlfiles/zwgkzt/ptjnj/ year2012/index2012.html. Accessed 25 Oct 2013.

3. Chen Z. Basic medical security systems cover $95 \%$ of the total population [in Chinese]. 2012 National Health Conference. 5 Jan 2012. http://money.163.com/12/0106/08/7N2Q8AN700253B0H. html. Accessed 25 Oct 2013.

4. Yang Q. 832 million people participate in NRCMS in China, participation rate exceeded $97 \%$. The Ministry of Health press conference [in Chinese]. 27 Feb 2012. http://finance.chinanews. com/jk/2012/02-27/3700114.shtml. Accessed 25 Oct 2013.

5. Chen Z, Mao Z. Ending the subsidization of medical services with profits from the sales of medicines and deepening the reform of public hospitals. 8 Oct 2012. http://english.qstheory.cn/society/ 201210/t20121009_185287.htm. Accessed 13 Nov 2013.

6. General Office of the State Council. Notice of the General Office of the State Council on issuing the work arrangements for the reform of the medical and health care system in five key aspects 
[in Chinese]. Beijing: General Office of the State Council; 2010. http://www.gov.cn/zwgk/2010-04/19/content_1586732.htm. Accessed 25 Oct 2013.

7. People's Daily Online. China issues new essential drug list. 16 Mar 2013. http://english.peopledaily.com.cn/90882/8170200. html. Accessed 13 Nov 2013.

8. National Health Development Research Center. Green book on China health development: research on healthcare reforms [in Chinese]. Beijing: People's Medical Publishing House; 2013.

9. Manning WG, Newhouse JP, Duan N, et al. Health insurance and the demand for medical care: evidence from a randomized experiment. Am Econ Rev. 1987;77(3):251-77.

10. Casalino LP, Nicholson S, Gans DN, et al. What does it cost physician practices to interact with health insurance plans? Health Aff (Millwood). 2009;28(4):w533-43.

11. Cutler DM, Sahni NR. If slow rate of health care spending growth persists, projections may be off by $\$ 770$ billion. Health Aff (Millwood). 2013;32(5):841-50.

12. Morgan SG, McMahon M, Mitton C, et al. Centralized drug review processes in Australia, Canada, New Zealand, and the United Kingdom. Health Aff (Millwood). 2006;25(2):337-47.

13. Liu GG, Eggleston $\mathrm{K}, \mathrm{Hu} \mathrm{T}$. Emerging health economics and outcomes research in the Asia-Pacific region. Value Health. 2008;11(Suppl 1):S1-2.

14. Liu G, Tang Y, Liu L. The urban employee medical insurance policy analysis: medical savings account and medical expenditures. Finance Econ. 2009;1(250):45-52.

15. Langley $\mathrm{PC}, \mathrm{Mu} \mathrm{R}, \mathrm{Wu} \mathrm{M}$, et al. The impact of rheumatoid arthritis on the burden of disease in urban China. J Med Econ. 2011;14(6):709-19.

16. Wang $\mathrm{Y}, \mathrm{Xu} \mathrm{K}, \mathrm{Hu} \mathrm{H}$, et al. Prevalence, risk factors, and impact on health related quality of life of overactive bladder in China. Neurourol Urodyn. 2011;30(8):1448-55.

17. Wang R, Wu M, Ma X, et al. Body mass index and health-related quality of life in adults: a population based study in five cities in China. Eur J Public Health. 2012;22(4):497-502.

18. Jing S, Yin A, Shi L, Liu J. Whether New Cooperative Medical Schemes reduce the economic burden of chronic disease in rural China. PLoS One. 2013;8(1):e53062.

19. Babiarz KS, Miller G, Yi H, et al. New evidence on the impact of China's New Rural Cooperative Medical Scheme and its implications for rural primary healthcare: multivariate difference-indifference analysis. BMJ. 2010;341:c5617.

20. Wang S, Peng L, Li J, et al. A trial-based cost-effectiveness analysis of erlotinib alone versus platinum-based doublet chemotherapy as first-line therapy for Eastern Asian nonsquamous non-small-cell lung cancer. PLoS One. 2013;8(3):e55917.

21. Wu B, Ye M, Chen H, Shen JF. Costs of trastuzumab in combination with chemotherapy for HER2-positive advanced gastric or gastroesophageal junction cancer: an economic evaluation in the Chinese context. Clin Ther. 2012;34(2):468-79.
22. Peking University, China Center for Health Economics Research. China guidelines for pharmacoeconomic evaluations, 9 Apr 2011. http://www.ispor.org/peguidelines/countrydet.asp?c=28\&t=4. Accessed 25 Oct 2013.

23. Wu EQ, Xie J, Wu C, Du EX, Li N, Tan R, et al. Treatment, monitoring, and economic outcomes of venous thromboembolism among hospitalized patients in China. PharmacoEconomics. 2014. doi:10.1007/s40273-014-0131-4

24. Wang Y, Wang Y, Qain Y, Zhang J, Tang X, Sun J, Zhu D. Association of body mass index with cause specific deaths in Chinese elderly hypertensive patients: Minhang community study. PLoS One. 2013;8(8):e71223.

25. Xue Q, Xiong X, Feng Y, Yao L, Chen S, Xiang L. Socioeconomic factors influencing anti psychotic prescription for schizophrenia inpatients in China: a cross-sectional study. Int Clin Psychopharmacol. Epub 2013 Dec 7.

26. Wu EQ, Wu J, Yang HY, Zheng Y, Xie K, Yu R, Xie J. Economic burden of hepatocellular carcinoma in China. Presented at the ISPOR 17th Annual International Meeting poster session, Washington, DC, 2012 Jun 2-6.

27. Xu J, Liu G, Deng G, Li L, Xiong X, Basu K. A comparison of outpatient healthcare expenditures between public and private medical institutions in urban China: an instrumental variable approach. Health Econ. Epub 2013 Dec 11.

28. Chen G, Liu GG, Xu F. The impact of the urban resident basic medical insurance on health services utilisation in China. PharmacoEconomics. 2014. doi:10.1007/s40273-013-0097-7

29. Chen M, Wang L, Chen W, Zhang L, Jiang H, Mao W. Does economic incentive matter for rational use of medicine? China's experience from the essential medicines program. PharmacoEconomics. 2014. doi:10.1007/s40273-013-0068-z

30. Wu J, Xu J, Liu GG, Wu J. Pharmaceutical pricing: an empirical study of market competition in Chinese hospitals. PharmacoEconomics. 2014. doi:10.1007/s40273-013-0099-5

31. Shi L, Yang HY, Cheng G, Meng Q. Time trends and determinants of pharmaceutical expenditure in China (1990-2009). PharmacoEconomics. 2014. doi:10.1007/s40273-013-0072-3

32. Yuan S, Liu Y, Li N, Zhang Y, Zhang Z, Tao J, et al. Impacts of health insurance benefit design on percutaneous coronary intervention use and inpatient costs among patients with acute myocardial infarction in Shanghai, China. PharmacoEconomics. 2014. doi:10.1007/s40273-013-0079-9

33. Chongqing T, Liubao P, Xiaohui Z, Jianhe L, Xiaomin W, Gannong C, et al. Cost-utility analysis of the newly recommended adjuvant chemotherapy for resectable gastric cancer patients in the 2011 Chinese National Comprehensive Cancer Network $(\mathrm{NCCN})$ clinical practice guidelines in oncology: gastric cancer. PharmacoEconomics. 2014. doi:10.1007/s40273-013-0065-2 